\title{
Mathematic model for describing the stress-tension behavior of an autoclave with integrated heating element
}

\author{
Denis Elovenko1), Alexander Graf2), Verena Kräusel2), Andreas Hirsch³)
}

1)Department of Design and Standardization in Mechanical Engineering, elovenko03@gmail.com; elovenkoda@mail.ru, Irkutsk National Research Technical University, 83, Lermontov street, 664074 Irkutsk, Russian Federation

2)Professorship for Forming and Joining, alexander.graf@mb.tu-chemnitz.de, verena.kraeusel@mb.tu-chemnitz.de, Chemnitz University of Technology, Reichenhainer Str. 70, 09107, Chemnitz, Germany

3) Professorship for Machine Tools and Forming Technology, wzm@mb.tu-chemnitz.de, Chemnitz University of Technology, Reichenhainer Str. 70, 09107, Chemnitz, Germany

\section{Keywords}

Contact Pressure, Displacement, Elongation, Modulus of Elasticity, Pressure, Thermal Field

\begin{abstract}
This paper describes a mathematical model and the method for determining the thermal stress fields in a cylindrical wall element of a pressure vessel with a built-in heating element and a layer of heat insulating material. The method presented is based on the calculation of contact pressures.
\end{abstract}

\section{Introduction}

Most pressure vessel (autoclave) designs have an internal heater. Their common disadvantage is the position of the heating elements in the reaction chamber. This reduces the usable volume of the reaction chamber and leads to a rapid failure of the heating elements. The same applies to the overheating of the supporting wall of the pressure vessel (autoclaves). This results in a reduction in its strength and the associated pressure that can be generated.

Multiple studies have been conducted regarding multilayer structures. The various circumferential layers with different properties of a solid propellant rocket motor are described here [1] to obtain an analytical solution for the general case of a multilayered thick cylindrical shell for internal pressure and thermal loads.

These previous studies consider the internal pressure exerted by both the cylindrical Ti-Al alloy liner and the carbon fiber resin composite (CFRC) wound layers, as shown by two models which have been built to illustrate the findings [2]. The first model is a cylinder loaded only with the internal pressure in the hoop direction. The second is a cylinder loaded only with the internal pressure in the axial direction. Solutions of stresses both in the hoop direction and in the axial direction were obtained by every layer loaded under internal pressure.

There is also a method for determining the transient heat transfer coefficient in cylindrical, thick-walled pressure parts [3]. The temperatures could be predicted at discrete locations throughout the wall if input data, such as thermocouple responses, was defined for one or more interior layers.

In a further publication, the stress state of laminated inhomogeneous closed cylindrical shells with an arbitrary cross-section was investigated, including transverse shear based on the linear element hypothesis [4]. The presented results were calculated for a two-layer cylindrical shell whose crosssection was a combination of an oval and a circle. 
The following paper presents a general and efficient stress analysis strategy for hollow composite cylindrical structures consisting of multiple layers of different anisotropic materials, which are subjected to different loads [5]. Cylindrical material anisotropy and various loading conditions were considered for the stress analysis. The stress analysis method was demonstrated with an example of a 22-layer composite riser and the results were compared with numerical solutions.

The construction of the cylindrical wall in Figure 1 is assumed to be ideal. If the outer layer 5 is larger than the remaining layers, it can be calculated by [6], otherwise the calculation must be performed using known analytical solutions [7, 8]. The inner wall (Figure 1, No. 1) is a highly stressed element and has a complex stress-strain state, which is directly influenced by the layer with the heating elements (Figure 1 , No. 2) by the parameters shape and position in the function as a spacer $[9,10]$. The thickness and material of the thermal insulation layer are determined by its thermal properties [11], which are intended to eliminate the reduction of the mechanical properties of the retaining wall (Figure 1, No. 5) due to undesired overheating during the operation of the autoclave.

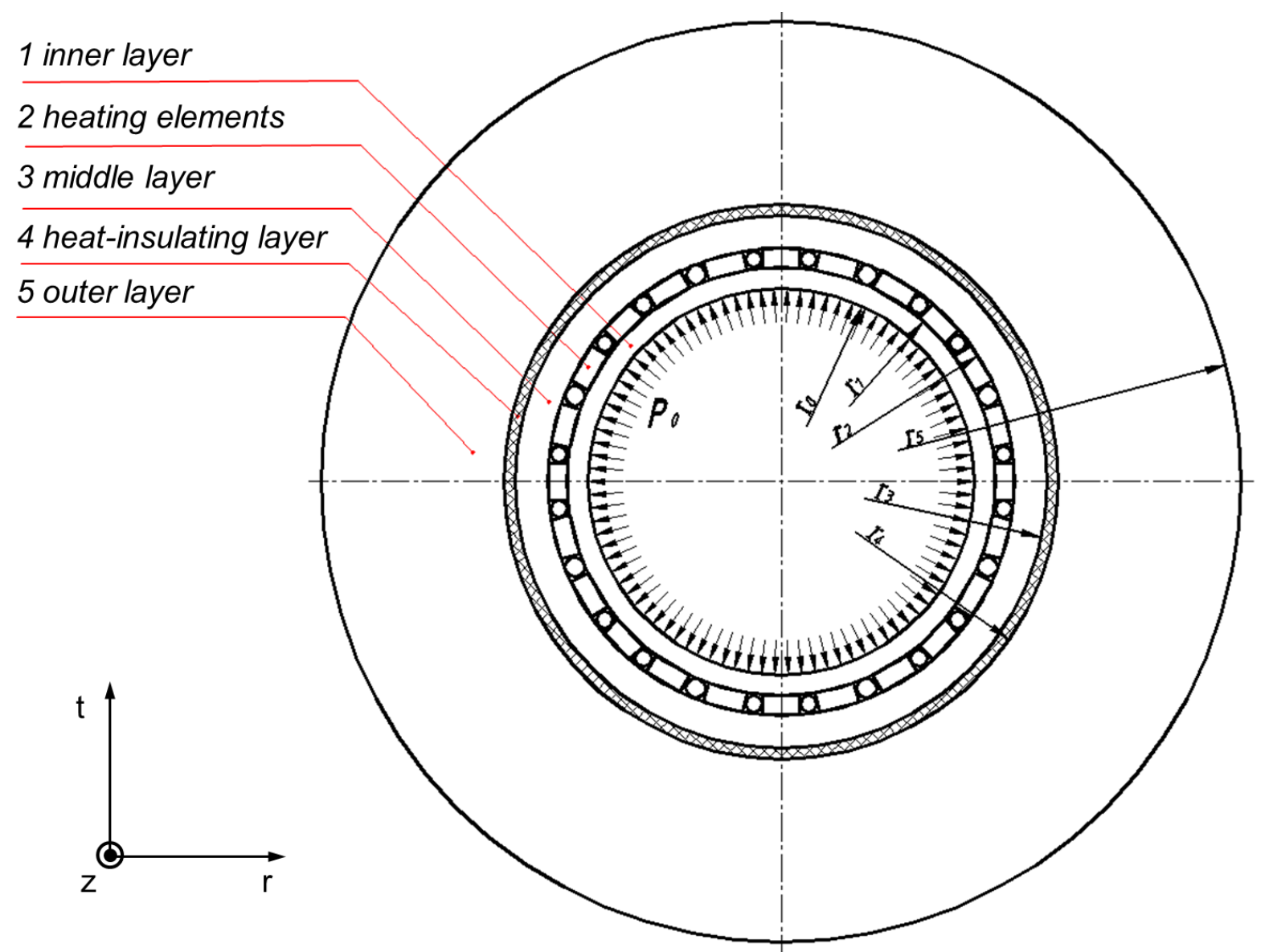

Figure 1: Structure diagram of the cylindrical wall of the high-pressure autoclave

\section{Determination of the contact pressure in the cylindrical wall of the pressure vessel}

The cylindrical wall of the autoclave (figure 1) is recognized as elastic. The main criterion for the elastic deformation under pressure is the fulfilment of the compatibility conditions for deformations of its contact surfaces. The conditions for the equality of the radial displacements $\left(u_{i}\left(r_{i}\right)\right)$ of the conjugated components of structure (figure 1, No. 1), where the layer formed by heating elements and spacers (Figure 11, No. 2) is assumed to be fixed, are generally determined by a system of equations. 


$$
\left\{\begin{array}{l}
u_{1}\left(r_{1}\right)=u_{2}\left(r_{1}\right) \\
u_{2}\left(r_{2}\right)=u_{3}\left(r_{2}\right) \\
u_{3}\left(r_{3}\right)=u_{4}\left(r_{3}\right) \\
u_{4}\left(r_{4}\right)=u_{5}\left(r_{4}\right)
\end{array} .\right.
$$

which under internal pressure $P_{0}$ has the general form

$$
\left\{\begin{array}{l}
\frac{r_{1}}{E_{1}}\left(\sigma_{t}^{1}\left(P_{0}\right)-\mu\left(\sigma_{r}^{1}\left(P_{0}\right)+\sigma_{z}^{1}\left(P_{0}\right)\right)\right)=\frac{r_{1}}{E_{2}}\left(\sigma_{t}^{2}\left(P_{0}\right)-\mu\left(\sigma_{r}^{2}\left(P_{0}\right)+\sigma_{z}^{2}\left(P_{0}\right)\right)\right) \\
\frac{r_{2}}{E_{2}}\left(\sigma_{t}^{2}\left(P_{0}\right)-\mu\left(\sigma_{r}^{2}\left(P_{0}\right)+\sigma_{z}^{2}\left(P_{0}\right)\right)\right)=\frac{r_{2}}{E_{3}}\left(\sigma_{t}^{3}\left(P_{0}\right)-\mu\left(\sigma_{r}^{3}\left(P_{0}\right)+\sigma_{z}^{3}\left(P_{0}\right)\right)\right) \\
\frac{r_{3}}{E_{3}}\left(\sigma_{t}^{3}\left(P_{0}\right)-\mu\left(\sigma_{r}^{3}\left(P_{0}\right)+\sigma_{z}^{3}\left(P_{0}\right)\right)\right)=\frac{r_{3}}{E_{4}}\left(\sigma_{t}^{4}\left(P_{0}\right)-\mu\left(\sigma_{r}^{4}\left(P_{0}\right)+\sigma_{z}^{4}\left(P_{0}\right)\right)\right) \\
\frac{r_{4}}{E_{4}}\left(\sigma_{t}^{4}\left(P_{0}\right)-\mu\left(\sigma_{r}^{4}\left(P_{0}\right)+\sigma_{z}^{4}\left(P_{0}\right)\right)\right)=\frac{r_{4}}{E_{5}}\left(\sigma_{t}^{5}\left(P_{0}\right)-\mu\left(\sigma_{r}^{5}\left(P_{0}\right)+\sigma_{z}^{5}\left(P_{0}\right)\right)\right)
\end{array} .\right.
$$

(explanation: $E_{i}$ - modulus of elasticity; $\sigma_{i}-$ tension $\left(\sigma_{r}\right.$ - radial tension; $\sigma_{t}-$ hoop tension; $\sigma_{z}-$ axial tension); $\mu_{i}$ - poisson's ratio)

In this system, the axial stresses are expressed as follows

$$
\sigma_{z}^{i}\left(P_{0}\right)=E_{i} \varepsilon_{z}+\mu\left(\sigma_{t}^{i}\left(P_{0}\right)+\sigma_{r}^{i}\left(P_{0}\right)\right) .
$$

(explanation: $\varepsilon_{z}$ - axial strain)

Then system (2) can be represented in the form 


$$
\left\{\begin{array}{l}
\frac{r_{1}}{E_{1}}\left(\sigma_{t}^{1}\left(P_{0}\right)-\mu\left(\sigma_{r}^{1}\left(P_{0}\right)+E_{1} \varepsilon_{z}+\mu\left(\sigma_{t}^{1}\left(P_{0}\right)+\sigma_{r}^{1}\left(P_{0}\right)\right)\right)\right)= \\
=\frac{r_{1}}{E_{2}}\left(\sigma_{t}^{2}\left(P_{0}\right)-\mu\left(\sigma_{r}^{2}\left(P_{0}\right)+E_{2} \varepsilon_{z}+\mu\left(\sigma_{t}^{2}\left(P_{0}\right)+\sigma_{r}^{2}\left(P_{0}\right)\right)\right)\right) \\
\frac{r_{2}}{E_{2}}\left(\sigma_{t}^{2}\left(P_{0}\right)-\mu\left(\sigma_{r}^{2}\left(P_{0}\right)+E_{2} \varepsilon_{z}+\mu\left(\sigma_{t}^{2}\left(P_{0}\right)+\sigma_{r}^{2}\left(P_{0}\right)\right)\right)\right)= \\
=\frac{r_{2}}{E_{3}}\left(\sigma_{t}^{3}\left(P_{0}\right)-\mu\left(\sigma_{r}^{3}\left(P_{0}\right)+E_{3} \varepsilon_{z}+\mu\left(\sigma_{t}^{3}\left(P_{0}\right)+\sigma_{r}^{3}\left(P_{0}\right)\right)\right)\right) \\
\frac{r_{3}}{E_{3}}\left(\sigma_{t}^{3}\left(P_{0}\right)-\mu\left(\sigma_{r}^{3}\left(P_{0}\right)+E_{3} \varepsilon_{z}+\mu\left(\sigma_{t}^{3}\left(P_{0}\right)+\sigma_{r}^{3}\left(P_{0}\right)\right)\right)\right)= \\
=\frac{r_{3}}{E_{4}}\left(\sigma_{t}^{4}\left(P_{0}\right)-\mu\left(\sigma_{r}^{4}\left(P_{0}\right)+E_{4} \varepsilon_{z}+\mu\left(\sigma_{t}^{4}\left(P_{0}\right)+\sigma_{r}^{4}\left(P_{0}\right)\right)\right)\right) \\
\frac{r_{4}}{E_{4}}\left(\sigma_{t}^{4}\left(P_{0}\right)-\mu\left(\sigma_{r}^{4}\left(P_{0}\right)+E_{4} \varepsilon_{z}+\mu\left(\sigma_{t}^{4}\left(P_{0}\right)+\sigma_{r}^{4}\left(P_{0}\right)\right)\right)\right)= \\
=\frac{r_{4}}{E_{5}}\left(\sigma_{t}^{5}\left(P_{0}\right)-\mu\left(\sigma_{r}^{5}\left(P_{0}\right)+E_{5} \varepsilon_{z}+\mu\left(\sigma_{t}^{5}\left(P_{0}\right)+\sigma_{r}^{5}\left(P_{0}\right)\right)\right)\right)
\end{array} .\right.
$$

Let us write this system explicitly

$$
\left\{\begin{array}{l}
\frac{\left(1-\mu^{2}\right)}{E_{1}}\left(\frac{P_{0}}{\beta_{1}^{2}-1}\left(1+\frac{r_{1}^{2}}{r_{1}^{2}}\right)+\left(-\frac{P_{1} \beta_{1}^{2}}{\beta_{1}^{2}-1}\left(1+\frac{r_{0}^{2}}{r_{1}^{2}}\right)\right)\right)-\frac{\left(\mu+\mu^{2}\right)}{E_{1}}\left(-P_{1}\right)= \\
=\frac{\left(1-\mu^{2}\right)}{E_{2}}\left(\frac{P_{1}}{\beta_{2}^{2}-1}\left(1+\frac{r_{2}^{2}}{r_{1}^{2}}\right)+\left(-\frac{P_{2} \beta_{2}^{2}}{\beta_{2}^{2}-1}\left(1+\frac{r_{1}^{2}}{r_{1}^{2}}\right)\right)\right)-\frac{\left(\mu+\mu^{2}\right)}{E_{2}}\left(-P_{1}\right) \\
\frac{\left(1-\mu^{2}\right)}{E_{2}}\left(\frac{P_{1}}{\beta_{2}^{2}-1}\left(1+\frac{r_{2}^{2}}{r_{2}^{2}}\right)+\left(-\frac{P_{2} \beta_{2}^{2}}{\beta_{2}^{2}-1}\left(1+\frac{r_{1}^{2}}{r_{2}^{2}}\right)\right)\right)-\frac{\left(\mu+\mu^{2}\right)}{E_{2}}\left(-P_{2}\right)= \\
=\frac{\left(1-\mu^{2}\right)}{E_{3}}\left(\frac{P_{2}}{\beta_{3}^{2}-1}\left(1+\frac{r_{3}^{2}}{r_{2}^{2}}\right)+\left(-\frac{P_{3} \beta_{3}^{2}}{\beta_{3}^{2}-1}\left(1+\frac{r_{2}^{2}}{r_{2}^{2}}\right)\right)\right)-\frac{\left(\mu+\mu^{2}\right)}{E_{3}}\left(-P_{2}\right) \\
\frac{\left(1-\mu^{2}\right)}{E_{3}}\left(\frac{P_{2}}{\beta_{3}^{2}-1}\left(1+\frac{r_{3}^{2}}{r_{3}^{2}}\right)+\left(-\frac{P_{3} \beta_{3}^{2}}{\beta_{3}^{2}-1}\left(1+\frac{r_{2}^{2}}{r_{3}^{2}}\right)\right)\right)-\frac{\left(\mu+\mu^{2}\right)}{E_{3}}\left(-P_{3}\right)=\cdot \\
=\frac{\left(1-\mu^{2}\right)}{E_{4}}\left(\frac{P_{3}}{\beta_{4}^{2}-1}\left(1+\frac{r_{4}^{2}}{r_{3}^{2}}\right)+\left(-\frac{P_{4} \beta_{4}^{2}}{\beta_{4}^{2}-1}\left(1+\frac{r_{3}^{2}}{r_{3}^{2}}\right)\right)\right)-\frac{\left(\mu+\mu^{2}\right)}{E_{4}}\left(-P_{3}\right) \\
\frac{\left(1-\mu^{2}\right)}{E_{4}}\left(\frac{P_{3}}{\beta_{4}^{2}-1}\left(1+\frac{r_{4}^{2}}{r_{4}^{2}}\right)+\left(-\frac{P_{4} \beta_{4}^{2}}{\beta_{4}^{2}-1}\left(1+\frac{r_{3}^{2}}{r_{4}^{2}}\right)\right)-\frac{\left(\mu+\mu^{2}\right)}{E_{4}}\left(-P_{4}\right)=\right. \\
=\frac{\left(1-\mu^{2}\right)}{E_{5}}\left(\frac{P_{4}}{\beta_{5}^{2}-1}\left(1+\frac{r_{5}^{2}}{r_{4}^{2}}\right)\right)-\frac{\left(\mu+\mu^{2}\right)}{E_{5}}\left(-P_{4}\right)
\end{array} .\right.
$$

(explanation: $\beta_{i}$ - coefficient thickness $\left(\beta_{i}=\frac{r_{i}}{r_{i-1}}\right)$ )

The system of equations (5) can be used to determine unknown contact pressures $P_{1}, P_{2}, P_{3}, P_{4}$. 
Due to the geometric parameters and physical properties of the materials, the system of equations (5) can be reduced to the form

$$
\left\{\begin{array}{l}
P_{1}\left(\left(\mu+\mu^{2}\right)\left(\frac{1}{E_{1}}-\frac{1}{E_{2}}\right)-\frac{\left(1-\mu^{2}\right)\left(r_{1}^{2}+r_{0}^{2}\right)}{E_{1}\left(r_{1}^{2}-r_{0}^{2}\right)}-\frac{\left(1-\mu^{2}\right)\left(r_{2}^{2}+r_{1}^{2}\right)}{E_{2}\left(r_{2}^{2}-r_{1}^{2}\right)}\right)+P_{2}\left(\frac{\left(1-\mu^{2}\right) 2 \beta_{2}^{2}}{E_{2}\left(\beta_{2}^{2}-1\right)}\right)=-P_{0}\left(\frac{\left(1-\mu^{2}\right) 2}{E_{1}\left(\beta_{1}^{2}-1\right)}\right) \\
P_{1}\left(\frac{\left(1-\mu^{2}\right) 2}{E_{2}\left(\beta_{2}^{2}-1\right)}\right)+P_{2}\left(\left(\mu+\mu^{2}\right)\left(\frac{1}{E_{2}}-\frac{1}{E_{3}}\right)-\frac{\left(1-\mu^{2}\right)\left(r_{2}^{2}+r_{1}^{2}\right)}{E_{2}\left(r_{2}^{2}-r_{1}^{2}\right)}-\frac{\left(1-\mu^{2}\right)\left(r_{3}^{2}+r_{2}^{2}\right)}{E_{3}\left(r_{3}^{2}-r_{2}^{2}\right)}\right)+P_{3}\left(\frac{\left(1-\mu^{2}\right) 2 \beta_{3}^{2}}{E_{3}\left(\beta_{3}^{2}-1\right)}\right)=0 \\
P_{2}\left(\frac{\left(1-\mu^{2}\right) 2}{E_{3}\left(\beta_{3}^{2}-1\right)}\right)+P_{3}\left(\left(\mu+\mu^{2}\right)\left(\frac{1}{E_{3}}-\frac{1}{E_{4}}\right)-\frac{\left(1-\mu^{2}\right)\left(r_{3}^{2}+r_{2}^{2}\right)}{E_{3}\left(r_{3}^{2}-r_{2}^{2}\right)}-\frac{\left(1-\mu^{2}\right)\left(r_{4}^{2}+r_{3}^{2}\right)}{E_{4}\left(r_{4}^{2}-r_{3}^{2}\right)}\right)+P_{4}\left(\frac{\left(1-\mu^{2}\right) 2 \beta_{4}^{2}}{E_{4}\left(\beta_{4}^{2}-1\right)}\right)=0 \\
P_{3}\left(\frac{\left(1-\mu^{2}\right) 2}{E_{4}\left(\beta_{4}^{2}-1\right)}\right)+P_{4}\left(\left(\mu+\mu^{2}\right)\left(\frac{1}{E_{4}}-\frac{1}{E_{5}}\right)-\frac{\left(1-\mu^{2}\right)\left(r_{4}^{2}+r_{3}^{2}\right)}{E_{4}\left(r_{4}^{2}-r_{3}^{2}\right)}-\frac{\left(1-\mu^{2}\right)\left(r_{5}^{2}+r_{4}^{2}\right)}{E_{5}\left(r_{5}^{2}-r_{4}^{2}\right)}\right)=0
\end{array}\right.
$$

and by a matrix method with respect to unknown $P_{1}, P_{2}, P_{3}, P_{4}$.

For structures (Figure 1) the integral state of equilibrium has the form

$$
\int_{r_{0}}^{r_{1}} \sigma_{t}^{1} d r+\int_{r_{1}}^{r_{2}} \sigma_{t}^{2} d r+\int_{r_{2}}^{r_{3}} \sigma_{t}^{3} d r+\int_{r_{3}}^{r_{4}} \sigma_{t}^{4} d r+\int_{r_{4}}^{r_{5}} \sigma_{t}^{5} d r=P_{0} r_{0}
$$

The layer with heating elements (figure 1, No. 2) is not continuous and does not perceive any ringshaped stresses (shear stress and tangential stress) as the layer of heat-insulating material (figure 1 , No. 4). Taking this into account, equation (7) has the form

$$
\int_{r_{0}}^{r_{1}} \sigma_{t}^{1}\left(P_{0}\right) d r+\int_{r_{2}}^{r_{3}} \sigma_{t}^{3}\left(P_{0}\right) d r+\int_{r_{4}}^{r_{5}} \sigma_{t}^{5}\left(P_{0}\right) d r=P_{0} r_{0},
$$

and including the formulas of Lamé, the equation will read as follows:

$$
\begin{aligned}
& \int_{r_{0}}^{r_{1}}\left(\frac{P_{0}}{\beta_{1}^{2}-1}\left(1+\frac{r_{1}^{2}}{r^{2}}\right)+\left(-\frac{P_{1} \beta_{1}^{2}}{\beta_{1}^{2}-1}\left(1+\frac{r_{0}^{2}}{r^{2}}\right)\right) d r+\int_{r_{2}}^{r_{3}}\left(\frac{P_{2}}{\beta_{3}^{2}-1}\left(1+\frac{r_{3}^{2}}{r^{2}}\right)+\left(-\frac{P_{3} \beta_{3}^{2}}{\beta_{3}^{2}-1}\left(1+\frac{r_{2}^{2}}{r^{2}}\right)\right) d r+\right.\right. \\
& +\int_{r_{4}}^{r_{5}} \frac{P_{4}}{\beta_{5}^{2}-1}\left(1+\frac{r_{5}^{2}}{r^{2}}\right) d r=P_{0} r_{0}
\end{aligned}
$$

If we integrate this expression, we get

$$
P_{2} r_{2}+P_{4} r_{4}=P_{1} r_{1}+P_{3} r_{3}
$$


For the structure under investigation, the expression (10) can be written in the form of a system

$$
\left\{\begin{array}{l}
P_{1} r_{1}=P_{2} r_{2} \\
P_{3} r_{3}=P_{4} r_{4}
\end{array} .\right.
$$

By adding the conditions for moving contact surfaces to this system, we obtain the system

$$
\left\{\begin{array}{l}
P_{1} r_{1}=P_{2} r_{2} \\
u_{1}\left(r_{1}\right)=u_{3}\left(r_{2}\right)+\left(\frac{\left(P_{1}+P_{2}\right)}{2}+\mu\left(\frac{P_{0}}{\beta^{2}-1}\right)\right) \frac{\left(r_{2}-r_{1}\right)}{E_{2}} \\
u_{3}\left(r_{3}\right)=u_{5}\left(r_{4}\right)+\left(\frac{\left(P_{3}+P_{4}\right)}{2}+\mu\left(\frac{P_{0}}{\beta^{2}-1}\right)\right) \frac{\left(r_{4}-r_{3}\right)}{E_{4}} \\
P_{3} r_{3}=P_{4} r_{4}
\end{array},\right.
$$

which is reduced to the form

$$
\left\{\begin{array}{l}
P_{1} r_{1}-P_{2} r_{2}=0 \\
\frac{r_{1}}{E_{1}}\left(\frac{P_{0}}{\beta_{1}^{2}-1}\left(1+\frac{r_{1}^{2}}{r_{1}^{2}}\right)+\left(-\frac{P_{1} \beta_{1}^{2}}{\beta_{1}^{2}-1}\left(1+\frac{r_{0}^{2}}{r_{1}^{2}}\right)\right)-\mu\left(\frac{P_{0}}{\beta^{2}-1}\right)-\mu\left(-P_{1}\right)\right)= \\
=\frac{r_{2}}{E_{3}}\left(\frac{P_{2}}{\beta_{3}^{2}-1}\left(1+\frac{r_{3}^{2}}{r_{2}^{2}}\right)+\left(-\frac{P_{3} \beta_{3}^{2}}{\beta_{3}^{2}-1}\left(1+\frac{r_{2}^{2}}{r_{2}^{2}}\right)\right)-\mu\left(\frac{P_{0}}{\beta^{2}-1}\right)-\mu\left(-P_{2}\right)\right)+\left(\frac{\left(P_{1}+P_{2}\right)}{2}+\mu\left(\frac{P_{0}}{\beta^{2}-1}\right)\right) \frac{\left(r_{2}-r_{1}\right)}{E_{2}} . \\
\frac{r_{3}}{E_{3}}\left(\frac{P_{2}}{\beta_{3}^{2}-1}\left(1+\frac{r_{3}^{2}}{r_{3}^{2}}\right)+\left(-\frac{P_{3} \beta_{3}^{2}}{\beta_{3}^{2}-1}\left(1+\frac{r_{2}^{2}}{r_{3}^{2}}\right)\right)-\mu\left(\frac{P_{0}}{\beta^{2}-1}\right)-\mu\left(-P_{3}\right)\right)= \\
=\frac{r_{4}}{E_{5}}\left(\frac{P_{4}}{\beta_{5}^{2}-1}\left(1+\frac{r_{5}^{2}}{r_{4}^{2}}\right)-\mu\left(\frac{P_{0}}{\beta^{2}-1}\right)-\mu\left(-P_{4}\right)\right)+\left(\frac{\left(P_{3}+P_{4}\right)}{2}+\mu\left(\frac{P_{0}}{\beta^{2}-1}\right)\right) \frac{\left(r_{4}-r_{3}\right)}{E_{4}} \\
P_{3} r_{3}-P_{4} r_{4}=0
\end{array} .\right.
$$

Assuming that all layers of the wall (figure 1) have the same modulus of elasticity (modulus of elasticity of the first kind), we obtain a system of equations for determining contact pressures $P_{1}, P_{2}, P_{3}, P_{4}$, taking into account the geometric characteristics of the wall structure and without using the modulus of elasticity of the layers. 


$$
\left\{\begin{array}{l}
P_{1} r_{1}-P_{2} r_{2}=0 \\
P_{1}\left(r_{1}\left(\mu-\frac{\left(r_{1}+r_{0}\right)}{\left(r_{1}-r_{0}\right)}\right)-\frac{\left(r_{2}-r_{1}\right)}{2}\right)-P_{2}\left(r_{2}\left(\mu+\frac{\left(r_{3}+r_{2}\right)}{\left(r_{3}-r_{2}\right)}\right)-\frac{\left(r_{2}-r_{1}\right)}{2}\right)+P_{3}\left(\frac{2 r_{2} \beta_{3}^{2}}{\left(\beta_{3}^{2}-1\right)}\right)= \\
=P_{0}\left(\frac{\mu\left(r_{1}-r_{2}\right)}{\beta^{2}-1}-\frac{2 r_{1}}{\left(\beta_{1}^{2}-1\right)}+\frac{\mu\left(r_{2}-r_{1}\right)}{\beta^{2}-1}\right) \\
P_{2}\left(\frac{2 r_{3}}{\left(\beta_{3}^{2}-1\right)}\right)+P_{3}\left(r_{3}\left(\mu-\frac{\left(r_{3}+r_{2}\right)}{\left(r_{3}-r_{2}\right)}\right)-\frac{\left(r_{4}-r_{3}\right)}{2}\right)-P_{4}\left(r_{4}\left(\mu+\frac{\left(r_{5}+r_{4}\right)}{\left(r_{5}-r_{4}\right)}\right)-\frac{\left(r_{4}-r_{3}\right)}{2}\right)= \\
=P_{0}\left(\frac{\mu\left(r_{3}-r_{4}\right)}{\beta^{2}-1}+\frac{\mu\left(r_{4}-r_{3}\right)}{\beta^{2}-1}\right) \\
P_{3} r_{3}-P_{4} r_{4}=0
\end{array}\right.
$$

The solution of the obtained system is also realized using the matrix method.

Using the equation systems (6) and (14), the modulus of elasticity for the layer formed by the heating elements can be calculated. It should be noted that the calculation of the modulus of elasticity $E_{2}$ in the system of equations (6) should be performed after the modulus of elasticity $E_{4}$ has been changed to the actual value of the modulus of elasticity of the thermal insulation material obtained by the experimental procedure. Then the actual values of the contact pressures that occurs in the structure (figure 1) loaded by the internal pressure $\mathrm{P}_{0}$ are determined.

\section{Distribution of the thermal field and consideration of its influence in determining the contact pressures in the cylindrical wall of a high-pressure vessel}

It has been demonstrated experimentally [14] that the maximum heating temperature in the wall takes place on the inner surface of the layer (figure 1, No. 3) behind the heating elements. The first two layers of the wall have the same heating temperature $T$, can be assumed to be evenly heated and have no temperature drop along their thickness.

In order to ensure the elastic operation function of the cylindrical wall in operating mode, it is necessary to reliably compress the heat-insulating layer, which is guaranteed to transfer radial loads to the supporting wall. The thermal expansion of layer 3 (figure 1) can cause the necessary compression of the thermal insulation layer, taking into account the material properties of this layer and the value of the thermal load in operating mode. Otherwise, the solution to this problem is to use metal for the central wall, whose coefficient of thermal expansion is higher by a than for the other metal components of the structure, which are equal $\alpha_{2}=\alpha_{3}=\alpha_{5}$. The coefficient of thermal expansion of thermal insulation materials is very low and their value is neglected $\alpha_{4}=0$. If we also consider the absence of radial stresses on the inner and outer surfaces of a solid cylindrical wall, whose thickness causes a temperature difference [12,13], we write down the compatibility conditions for deformations (4) taking into account the remarks made. 


$$
\left\{\begin{array}{l}
\frac{r_{1}}{E_{1}}\left(\left(1-\mu^{2}\right) \sigma_{t}^{1}\left(P_{0}\right)-\left(\mu+\mu^{2}\right) \sigma_{r}^{1}\left(P_{0}\right)-\mu E_{1} \varepsilon_{z}\right)+r_{1} \alpha_{1} T\left(r_{1}\right)= \\
=\frac{r_{1}}{E_{2}}\left(\left(1-\mu^{2}\right) \sigma_{t}^{2}\left(P_{0}\right)-\left(\mu+\mu^{2}\right) \sigma_{r}^{2}\left(P_{0}\right)-\mu E_{2} \varepsilon_{z}\right)+r_{1} \alpha_{2} T\left(r_{1}\right) \\
\frac{r_{2}}{E_{2}}\left(\left(1-\mu^{2}\right) \sigma_{t}^{2}\left(P_{0}\right)-\left(\mu+\mu^{2}\right) \sigma_{r}^{2}\left(P_{0}\right)-\mu E_{2} \varepsilon_{z}\right)+r_{2} \alpha_{2} T\left(r_{2}\right)= \\
=\frac{r_{2}}{E_{3}}\left(\left(1-\mu^{2}\right) \sigma_{t}^{3}\left(P_{0}, T_{0}\right)-\left(\mu+\mu^{2}\right) \sigma_{r}^{3}\left(P_{0}\right)-\mu E_{3} \varepsilon_{z}\right)+r_{2} \alpha_{3} T\left(r_{2}\right) \\
\frac{r_{3}}{E_{3}}\left(\left(1-\mu^{2}\right) \sigma_{t}^{3}\left(P_{0}, T_{0}\right)-\left(\mu+\mu^{2}\right) \sigma_{r}^{3}\left(P_{0}\right)-\mu E_{3} \varepsilon_{z}\right)+r_{3} \alpha_{3} T\left(r_{3}\right)= \\
=\frac{r_{3}}{E_{4}}\left(\left(1-\mu^{2}\right) \sigma_{t}^{4}\left(P_{0}\right)-\left(\mu+\mu^{2}\right) \sigma_{r}^{4}\left(P_{0}\right)-\mu E_{4} \varepsilon_{z}\right) \\
\frac{r_{4}}{E_{4}}\left(\left(1-\mu^{2}\right) \sigma_{t}^{4}\left(P_{0}\right)-\left(\mu+\mu^{2}\right) \sigma_{r}^{4}\left(P_{0}\right)-\mu E_{4} \varepsilon_{z}\right)= \\
=\frac{r_{4}}{E_{5}}\left(\left(1-\mu^{2}\right) \sigma_{t}^{5}\left(P_{0}, T_{0}\right)-\left(\mu+\mu^{2}\right) \sigma_{r}^{5}\left(P_{0}\right)-\mu E_{5} \varepsilon_{z}\right)+r_{4} \alpha_{5} T\left(r_{4}\right)
\end{array} .\right.
$$

(explanation: $\alpha_{i}$ - coefficient of linear expansion, $T$ - temperature)

The same system is in explicit form with respect to unknown contact pressures: 


$$
\left\{\begin{array}{l}
P_{1}\left(\left(\mu+\mu^{2}\right)\left(\frac{1}{E_{1}}-\frac{1}{E_{2}}\right)-\frac{\left(1-\mu^{2}\right)\left(r_{1}^{2}+r_{0}^{2}\right)}{E_{1}\left(r_{1}^{2}-r_{0}^{2}\right)}-\frac{\left(1-\mu^{2}\right)\left(r_{2}^{2}+r_{1}^{2}\right)}{E_{2}\left(r_{2}^{2}-r_{1}^{2}\right)}\right)+P_{2}\left(\frac{\left(1-\mu^{2}\right) 2 \beta_{2}^{2}}{E_{2}\left(\beta_{2}^{2}-1\right)}\right)= \\
=-P_{0}\left(\frac{\left(1-\mu^{2}\right) 2}{E_{1}\left(\beta_{1}^{2}-1\right)}\right)+\left(\alpha_{2}-\alpha_{1}\right) T_{0} \\
P_{1}\left(\frac{\left(1-\mu^{2}\right) 2}{E_{2}\left(\beta_{2}^{2}-1\right)}\right)+P_{2}\left(\left(\mu+\mu^{2}\right)\left(\frac{1}{E_{2}}-\frac{1}{E_{3}}\right)-\frac{\left(1-\mu^{2}\right)\left(r_{2}^{2}+r_{1}^{2}\right)}{E_{2}\left(r_{2}^{2}-r_{1}^{2}\right)}-\frac{\left(1-\mu^{2}\right)\left(r_{3}^{2}+r_{2}^{2}\right)}{E_{3}\left(r_{3}^{2}-r_{2}^{2}\right)}\right)+P_{3}\left(\frac{\left(1-\mu^{2}\right) 2 \beta_{3}^{2}}{E_{3}\left(\beta_{3}^{2}-1\right)}\right)= \\
=\frac{\alpha_{3}(1+\mu)\left(T_{0}-T\left(r_{3}\right)\right)}{2}\left(\frac{1}{\ln \left(\frac{r_{3}}{r_{2}}\right)}-\frac{\left.2\left(\frac{r_{3}}{r_{0}}\right)^{2}\right)}{\left.\left(\frac{r_{3}}{r_{0}}\right)^{2}-\left(\frac{r_{2}}{r_{0}}\right)^{2}\right)^{2}}\left(\frac{1}{E_{3}}-\frac{1}{E_{4}}\right)-\frac{\left(1-\mu^{2}\right)\left(r_{3}^{2}+r_{2}^{2}\right)}{E_{3}\left(r_{3}^{2}-r_{2}^{2}\right)}-\frac{\left(1-\mu^{2}\right)\left(r_{4}^{2}+r_{3}^{2}\right)}{E_{4}\left(r_{4}^{2}-r_{3}^{2}\right)}\right)+P_{4}\left(\frac{\left(1-\mu^{2}\right) 2 \beta_{4}^{2}}{E_{4}\left(\beta_{4}^{2}-1\right)}\right)=. \\
P_{2}\left(\frac{\left(1-\mu^{2}\right) 2}{E_{3}\left(\beta_{3}^{2}-1\right)}\right)+P_{3}\left(\left(\mu+\mu^{2}\right)\right. \\
\left.=-\alpha_{3} T_{0}-\frac{\alpha_{3}(1+\mu)\left(T_{0}-T\left(r_{3}\right)\right)}{2}\left(\frac{1}{\ln \left(\frac{r_{3}}{r_{2}}\right)}-\frac{\left.2\left(\frac{r_{2}}{r_{0}}\right)^{2}\right)}{\left.\left(\frac{r_{3}}{r_{0}}\right)^{2}-\left(\frac{r_{2}}{r_{0}}\right)^{2}\right)}\right)_{P_{3}\left(\frac{\left(1-\mu^{2}\right) 2}{E_{4}\left(\beta_{4}^{2}-1\right)}\right)+P_{4}\left(\left(\mu+\mu^{2}\right)\left(\frac{1}{E_{4}}-\frac{1}{E_{5}}\right)-\frac{\left(1-\mu^{2}\right)\left(r_{4}^{2}+r_{3}^{2}\right)}{E_{4}\left(r_{4}^{2}-r_{3}^{2}\right)}-\frac{\left(1-\mu^{2}\right)\left(r_{5}^{2}+r_{4}^{2}\right)}{E_{5}\left(r_{5}^{2}-r_{4}^{2}\right)}\right)=}^{2\left(\frac{r_{5}}{r_{0}}\right)^{2}-\left(\frac{r_{4}}{r_{0}}\right)^{2}}\right) \\
=\alpha_{5} T\left(r_{4}\right)+\frac{\alpha_{5}(1+\mu)\left(T\left(r_{4}\right)-T\left(r_{5}\right)\right)}{2}\left(\frac{1}{r_{0}}\right)
\end{array}\right.
$$

The thermal field coming from the heating elements in the cylindrical collector wall of the autoclave (figure 1 ) is axially symmetrical and the heat flow will be stationary along the wall thickness from the heating elements to the outer surface of the co-vessel. It can be described as:

$$
q_{l}=\frac{2 \pi\left(T_{0}-T_{\mathrm{env}}\right)}{\frac{1}{\lambda_{3}} \ln \left(\frac{r_{3}}{r_{2}}\right)+\frac{1}{\lambda_{4}} \ln \left(\frac{r_{4}}{r_{3}}\right)+\frac{1}{\lambda_{5}} \ln \left(\frac{r_{5}}{r_{4}}\right)+\frac{1}{K_{\mathrm{env}} r_{5}}}
$$

(explanation: $\lambda_{i}$ - thermal conductivity, $\mathrm{T}_{e n v}$ - ambient temperature, $\frac{1}{K_{e n v}}$ - ambient thermal resistance) Then, the unknown values of the temperatures at the contact radii in the system (16) can be calculated from the equations

$$
T\left(r_{3}\right)=T_{0}-\frac{q_{l}}{2 \pi} \frac{1}{\lambda_{3}} \ln \left(\frac{r_{3}}{r_{2}}\right),
$$




$$
\begin{aligned}
& T\left(r_{4}\right)=T\left(r_{3}\right)-\frac{q_{l}}{2 \pi} \frac{1}{\lambda_{4}} \ln \left(\frac{r_{4}}{r_{3}}\right), \\
& T\left(r_{5}\right)=T\left(r_{4}\right)-\frac{q_{l}}{2 \pi} \frac{1}{\lambda_{5}} \ln \left(\frac{r_{5}}{r_{4}}\right) .
\end{aligned}
$$

(explanation: $q_{l}$ - thermal flux)

The system of equations (16) is calculated using the matrix method and allows the determination of the values of the resulting contact pressures in the construction (figure 1), which are caused by the effects of the internal pressure and the temperature from the heating elements.

\section{The design parameters of the cylindrical walls of a high-pressure autoclave}

The composite cylindrical wall of a high-pressure autoclave (figure 1) will function correctly if the equivalent stresses in the structure do not exceed the permissible stresses of the material of one of its structural elements. Next, we will look at structures where the coefficients of thermal expansion of the steel elements do not differ from each other.

The condition for structural strength (figure 1 ) is

$$
\sigma_{\text {equ }}\left(r_{0}\right) \leq[\sigma]_{1}
$$

Equivalent stresses $\left(\sigma_{\text {equ }}\left(r_{0}\right)\right)$ on the inner surface of the cylindrical wall (figure 1, No. 1) according to the fourth strength theory have the form

$$
\sigma_{\text {equ }}\left(r_{0}\right)=\sqrt{\frac{\left(\sigma_{t}^{r_{0}}\left(P_{0}, T_{0}\right)-\sigma_{z}^{r_{0}}\left(P_{0}, T_{0}\right)\right)^{2}+\left(\sigma_{t}^{r_{0}}\left(P_{0}, T_{0}\right)+\sigma_{r}^{r_{0}}\left(P_{0}, T_{0}\right)\right)^{2}+\left(\sigma_{z}^{r_{0}}\left(P_{0}, T_{0}\right)+\sigma_{r}^{r_{0}}\left(P_{0}, T_{0}\right)\right)^{2}}{2}},
$$

and permissible stresses in the selection of the material of the structural element (figure 1, No. 1) are determined by the pressure exerted by the component of the composite wall structure

$$
[\sigma]_{1}=\frac{P_{0}}{\beta_{1}}
$$

In the design under consideration, the ring stresses determined by formula (18), taking into account the constant temperature of the central wall on the inner wall surface $r_{0}$, have the form 


$$
\sigma_{t}\left(r_{0}\right)=\frac{\left(P_{0}-P_{1}\right)\left(\frac{r_{1}}{r_{0}}\right)^{2}}{\left(\frac{r_{1}}{r_{0}}\right)^{2}-1}-\frac{\left(P_{1}\left(\frac{r_{1}}{r_{0}}\right)^{2}-P_{0}\right)}{\left(\frac{r_{1}}{r_{0}}\right)^{2}-1},
$$

and the axial stresses are represented by the following formula

$$
\sigma_{z}\left(r_{0}\right)=E_{1} \varepsilon_{z}+\mu\left(\sigma_{t}\left(r_{0}\right)+\sigma_{r}\left(r_{0}\right)\right)-\alpha_{1} E_{1} T_{0}
$$

In equation (25), axial strain has the form

$$
\varepsilon_{z}=\frac{(1-2 \mu)\left(P_{0} r_{0}^{2}\right)+\sum_{i=1}^{5}\left(\alpha_{i} E_{i}\right)\left(T_{i} r_{i}^{2}-T_{i-1} r_{i-1}^{2}+\left(T_{i-1}-T_{i}\right) \frac{\left(r_{i}^{2}-r_{i-1}^{2}\right)}{2 \ln \left(\frac{r_{i}}{r_{i-1}}\right)}\right)}{\sum_{i=1}^{5} E_{i}\left(r_{i}^{2}-r_{i-1}^{2}\right)} .
$$

The geometric parameters and properties of the materials of the cylindrical wall components, the heating temperature and the internal pressure are functions of the stress-strain state of the prefabricated cylinder wall of the autoclave. The stress intensity condition for determining optimum design parameters (figure 1) has the general form

$$
\sigma_{i}=\sigma_{i}\left(r_{0}, r_{1}, r_{2}, r_{3}, r_{4}, r_{5}, \beta, \lambda_{T I}, E_{T I}, P_{0}, T_{0}\right)-\frac{P_{0}}{\beta}=0
$$

$\lambda_{T I}-$ thermal conductivity of the thermal insulation layer

$E_{T I}$-modulus of elasticity of the thermal insulation layer

The models obtained make it possible to determine the optimum parameters of the cylindrical wall construction (figure 1) for high-pressure vessels with built-in heating elements and a layer of heatinsulating material.

The results of the elastic modulus calculation for the thermal insulation of chamotte powder (21 $738 \mathrm{MPa})$ under elastic work and under technological load and asbestos (19 $833 \mathrm{MPa}$ ) are considered. And from the research results [11] we know that these materials have a thermal conductivity between $0.77-1.411$. We can construct curves to determine the parameters for functional constructions.

The stress-strain condition of the inner wall (figure 1, No. 1) depends essentially on the thickness and properties of the thermal insulation. The modulus of elasticity of the thermal insulation set is assumed to be $20000 \mathrm{MPa}$. Temperature $\left(T_{0}\right)$ and internal pressure $\left(P_{0}\right)$ are the parameters of the technological (reaction) environment. For clarification the calculated curves were presented in this coordinate system $\left(P_{0}, T_{0}\right)$. Figure 2 a shows the graphic dependencies (curves) of the internal pressure from the heating 
element temperature for the thermal conductivity coefficients of the thermal insulation material with the parameters - $r_{0}=195 ; r_{1}=210 ; r_{2}=220 ; r_{3}=250 ; r_{4}=255 ; r_{5}=480 \mathrm{~mm} ; E_{1}=200000 ; E_{2}=33333.3$; $E_{3}=200000 ; E_{4}=20000 ; E_{5}=200000 \mathrm{MPa}$. And figure $2 \mathrm{~b}$ shows similar curves for construction with parameters $r_{0}=400 ; r_{1}=410 ; r_{2}=452 ; r_{3}=470 ; r_{4}=485 ; r_{5}=800 \mathrm{~mm} ; E_{1}=200000 ; E_{2}=40000 ; E_{3}$ $=200000 ; E_{4}=20000 ; E_{5}=200000 \mathrm{MPa}$.

a)

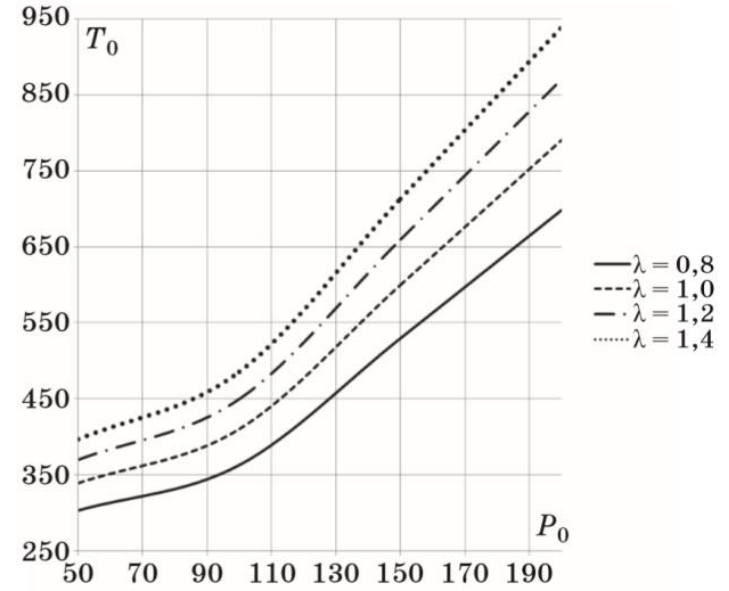

b)

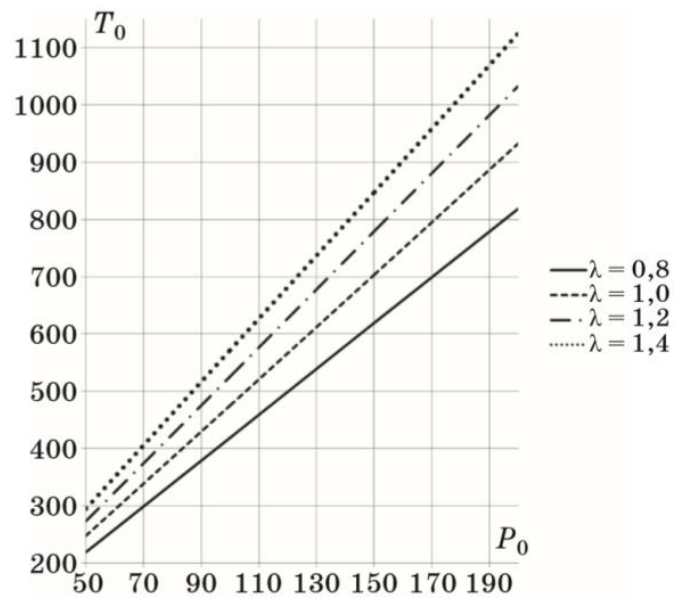

Figure 2: Curves for determining the central walls and heat insulation materials for the autoclave with integrated heating element

\section{Results}

The mathematical model and the method for determining thermal stress fields in a cylindrical wall element of a pressure vessel with a built-in heating element and a layer of heat insulating material was discussed. The method is based on the calculation of contact pressures. Contact pressures are calculated with regard to the condition of compatibility of the deformations of contacting layers.

\section{References}

[1] Renganathan K.; Nageswara Rao B.; Jana M.K.: A multilayered thick cylindrical shell under internal pressure and thermal loads applicable to solid propellant rocket motors. Forschung im Ingenieurwesen, 66 (2000) 2, pp. 57-66.

[2] Chuan-xiang Zheng.; Fan YangAi-shi Zhu: Mechanical analysis and reasonable design for Ti-Al alloy liner wound with carbon fiber resin composite high pressure vessel. Journal of Zhejiang University, 10 (2009) 3, pp. 384-391.

[3] Taler J.: Prediction of transient heat transfer coefficients in thick-walled pressure components of cylindrical geometry. Wärme - und Stoffübertragung, 25 (1990) 2, pp. 77-83.

[4] Bykhovets O.N.: Refined analysis of the influence of the inhomogeneous layers on the stress state of circular and noncircular cylindrical shells. International Applied Mechanics, 38 (2002) 7 , pp. 872-877.

[5] Sun X. S.; Tan V. B. C.; Chen Y.; Tan L. B.; Jaiman R. K.; Tay T. E.: Stress analysis of multilayered hollow anisotropic composite cylindrical structures using the homogenization method. Acta Mechanica, 255 (2014) 6, pp. 1649-1672.

[6] Elovenko D.: Development of a program for the calculation of temperature fields and stresses in a multilayer wall of a high-pressure tank. Application of mathematical methods and information 
technologies in the economy: Collection of scientific works - Irkutsk: Publishing BNUEL (2010), pp. 11-19.

[7] Kontorovich Z.: Basics of the calculation of chemical machines and apparatuses. Moscow: Publishing house MASHGIZ (1960), p. 744.

[8] Timoschenko S.; Goodyear J.: The theory of elasticity. Moscow: The science, (1979), p. 560

[9] Elovenko D.; Repetsky O.: Investigation of the influence of the distance bands of heating elements and their design parameters on the stressed condition of the middle wall in new highpressure autoclaves. Scholarship holders' bulletin DAAD, 1 (8), 2011, pp. 25-27.

[10] Elovenko D.; Repetsky O.: For the efficient design of distance strips of heating elements in highpressure autoclaves. Baikal research journal. 5, 2011. http://brjbguep.ru/reader/article.aspx?id=9724. (accessed 17 May 2018).

[11] Elovenko D.; Repetsky O.: Investigation of the thermophysical properties of thermal insulation materials for new constructions of cylindrical walls of high-pressure autoclaves. Bulletin of baikal state university. 6 (80), 2011, pp. 201-206. http://izvestia.bgu.ru/reader/article.aspx?id=14609. (accessed 17 May 2018).

[12] Birger I.: Strength, stability, vibrations. Reference work in three volumes. Moscow: The Mechanical engineering. 1979.

[13] Kovalenko A.: Fundamentals of thermoelasticity. Kiev: Naukova Dumka, 1970.

[14] Elovenko D.; Pimshtein P.; Repetsky O.; Tatarinov D.: Experimental investigation of the autoclave model for the hydrothermal synthesis of minerals. Bulletin of the Baikal Union of DAAD scholarship holders 1 (7), 2010, pp. 11-19. 